\title{
Computational Fluid Dynamics (CFD) use in the simulation of the death end ventilation in tunnels and galleries
}

\author{
J. Toraño, R. Rodríguez \& I. Diego \\ GIMOC, Mining Engineering and Civil Works Research Group, \\ Oviedo School of Mines, University of Oviedo, Oviedo, Spain
}

\begin{abstract}
In the framework of the Research Project CTM2005-00187/TECNO, "Prediction models and prevention systems in the particle atmospheric contamination in an industrial environment" of the Spanish National R $+\mathrm{D}$ Plan of the Ministry of Education and Science, 2004-2007 period, a CFD model has been developed to simulate air flows in tunnels and galleries, including its detailed comparison with available experimental data. Taking into account the importance of the air velocity distribution in the tunnels or galleries where there is continuous transport of particulated material by means of conveyor belts, and where PM10 or PM50 particulated material can be easily thrown in suspension, we quickly identify the necessity of studying the air behaviour using CFD software. Several models were developed using the commercial code Ansys CFX 10.0, starting from several 3D meshes of different resolutions generated using ICEM CFD 10.0. Medium complexity turbulence models were selected in order to obtain acceptable resolution times in single processor machines, as well as following advices contained in related bibliography. Zero Equation (constant turbulent eddy viscosity), k-epsilon and Spallart-Allmaras models were used, comparing their results with detailed experimental measurements obtained from the use of hot wire anemometers. Results show good agreement between the simulated data and the experimental measurements depending on the turbulence model: areas close to the tunnel end are best simulated using k-e, but areas far from the tunnel end, in the developed flow area, are best simulated using Spalart-Allmaras. As was expected the constant turbulent eddy viscosity model gave results which were not very approximated.
\end{abstract}

Keywords: death end ventilation, particulated material, CFD, turbulence. 


\section{Introduction}

Taking into account the importance of the velocity distribution in the ventilation of the tunnels or galleries where there is continuous transport of particulated material using conveyor belts, from which there can easily appear PM10 and PM50 airborne dust, it was clearly shown the necessity of studying the air flow behaviour through a state of the art tool, CFD or computational Fluid dynamics.

Among the hundreds of data that has to be introduced to start a CFD simulation one of the most important is the selection of the method that is going to perform the turbulence modelling. Generally speaking the model is selected depending on the technician expertise, the help of the CFD software support service, or related bibliography, [2], [3] or [7] in our case. This paper shows the use of three relative simple turbulence models that allow its future use in extensive meshes of complex geometries or multiphase modelling.

The knowledge acquired with these simulations will allow setting the bases for the subsequent simulations that will include dust behaviour. The dust movement will be checked against experimental measurements done using optical dust samplers, as was previously done by the research team that has developed this study, e.g. Toraño et al [5] and [6].

\section{Measurements in an disused mine gallery}

Measurements have been done in a death end gallery in a disused mine area. It is a gallery supported by a 2UA type roof support, approximately 72 meters ahead from the axis of the main gallery from it is started. Gallery is curved slightly to the right.
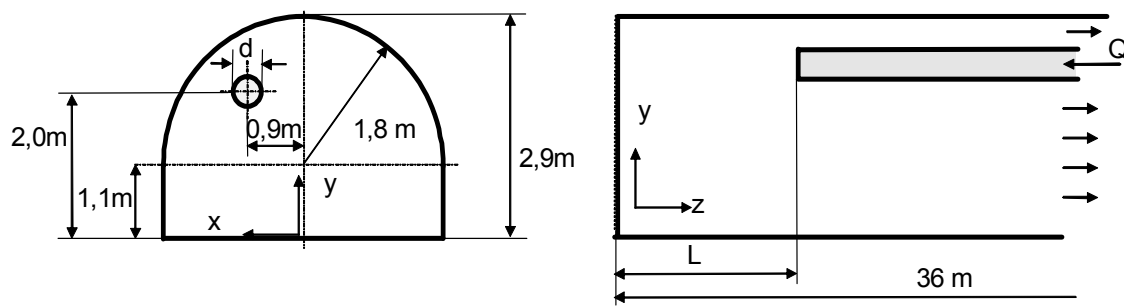

Figure 1: $\quad$ Draft of the disused mine gallery.

During the experiment the air was blown to the gallery end through a ventilation duct, using the power of an electric fan. The duct is flexible in its final part and goes parallel to the gallery wall. Its diameter is $600 \mathrm{~mm}$ and finishes its run 6 meters from the gallery end. Environmental conditions during the tests where $22-23^{\circ} \mathrm{C}$ and $75-80 \%$ of humidity.

Air velocity measurements where done with a hot wire anemometer, TSI IFA 100. Two different probes were used, one omnidirectional (measures the velocity under $0,8 \mathrm{~m} / \mathrm{s}$ ) and a film probe (measures the velocity module within 0 to $20 \mathrm{~m} / \mathrm{s}$ ). There were done around 50 measurements in each one of the gallery 
cross sections studied, and a total number of 16 cross sections were studied. This means a number of velocity measurement points as high as 800 . Estimated precision in the velocity measurement is around $+-5 \%$.

Measured data were shown and empirically treated in the development of a $\mathrm{PhD}$ of the University of Oviedo, see [8].

\section{CFD simulation}

The CFD commercial code Ansys CFX was used to develop a simulation model that allows the comparison of the experimental results and the simulated ones. The model considers a straight gallery and a section approximately semicircular, quite similar to the one that accommodated the experimental measurements.

\subsection{Geometry}

The simulated gallery has an approximate cross section of $9 \mathrm{~m}^{2}$ and an overall length of $36 \mathrm{~m}$. In this simulation there has been considered a ventilation pipe of $600 \mathrm{~mm}$ of diameter, that guides an airflow of $3,39 \mathrm{~m}^{3} / \mathrm{s}$. The $3 \mathrm{D}$ Geometry has been done with the software Solidworks.

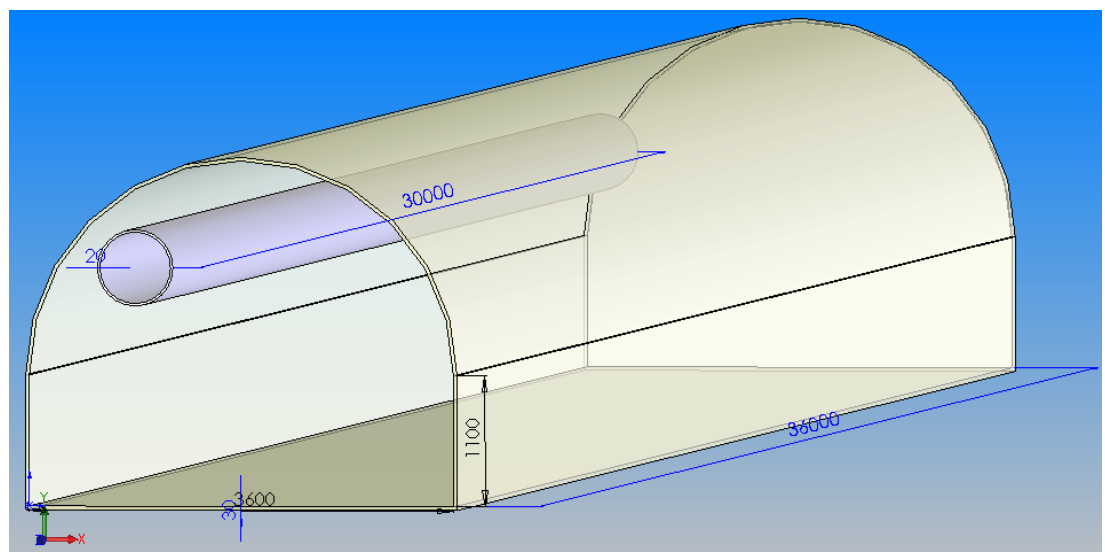

Figure 2: $\quad 3 \mathrm{D}$ geometry of the model.

On top of the surfaces needed to create the model, other auxiliary surfaces were created for a better subsequent meshing: one in the outlet of the gallery, the input and output covers of the ventilation pipe and an intermediate surface that will serve as divide the air in two regions with different meshing sizes.

\subsection{Meshing}

The meshing has been done using commercial software ANSYS ICEM CFD 10.0, importing the geometry from Solidworks through IGES format. First a geometry cleaning is done, deleting unnecessary geometry features and checking for physical incongruence. 
The mesh was done using tetrahedrical and prismatic cells, and after several tests and initial CFD calculations it was seen that there were not significantly differences in the calculation using meshes over 500k elements.

The mesh was not easy to develop, even using "fool-proof" software as ICEM, due to difficulties selecting appropriate mesh sizes to solve curved surfaces and the space between the pipe and the wall.

The mesh finally used will be different depending on the model area, coarse mesh of $500 \mathrm{~mm}$ of tetra size far from the gallery end, a fine mesh of $250 \mathrm{~mm}$ of tetra size near the gallery end and three times less in the ventilation tube area.

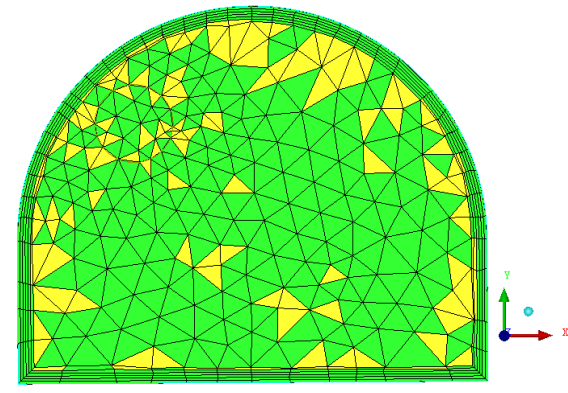

Figure 3: Meshing.

Walls will be meshed using prisms. After finishing the mesh and its optimization we finally get a mesh of $634 \mathrm{k}$ elements (shown in figure 3 ).

\subsection{CFD simulation}

\subsubsection{Introduction}

The physical characteristics of the problem are defined in CFX-Pre. Three different simulations will be done, depending on the turbulence model used: Zero equation, K-epsilon and Spallart-Allmaras. CFX offers a wide selection of turbulence models, from the quite simple Zero Equation models to LES and RANS, highly demanding in computing time and hardware requirements. The selection of these 3 more or less complex models is based on related bibliography, e.g. [2], [3] and [4].

\subsubsection{Resolution conditions}

Problem will be solved as stationary, with three domains separated by "domain interfaces" corresponding to the ventilation pipe, fine mesh and coarse mesh, and isothermal conditions at $23^{\circ} \mathrm{C}$

The boundary conditions used are:

- "Wall", with default characteristics and smooth roughness, located in all walls.

- "Outlet", located in the gallery air outlet, defined by a condition of "Average Static Pressure 0 atm". This air outlet could be also defined as an opening, but convergence behaviour seen with all simulations showed no recirculation presence at this plane. 
- "Inlet": characterized by the velocity obtained from the theoretical operating point of the fan installed in the experiment. Turbulence will be considered "medium", intensity $=5 \%$.

Convergence is set to reach a $1 \mathrm{E}-05$ level in all governing equations, which is accurate enough as per CFX support documentation.

In case of the "Zero Equation" turbulence model, and after several tests, the Eddy Length Scale is set at $1.25 \mathrm{~m}$.

The Spallart-Allmaras model is experimentally implemented in CFX 10.0, and CCL, CFX Command Language, must to be used to be able to start this model, as it is not available in the CFX-Pre GUI (Graphic User Interface).

\section{Results comparison}

Each one of the simulations done is compared with the existent experimental data. Comparisons are resumed in two ways: first a general flow comparison and secondly a detailed one in several remarkable sections.

\subsection{General view of flow}

Figure 4 shows simultaneously cut planes for the velocity flow field, representing velocity contours in following planes: $X Y$ at $0.5,8,18$ and 29 meters from the gallery end, $\mathrm{ZX}$ at 0.4 meters from bottom and $\mathrm{YZ}$ at $0.5 \mathrm{~m}$ from left in the outflow adventional sense. (As seen in the figure, $Z$ is aligned with the gallery axis, $\mathrm{Y}$ is the vertical and $\mathrm{X}$ is the cross direction axis).

The airflow is mainly characterized by its turn in the middle of the studied domain, as well as its highly turbulent behaviour near the gallery end, with an area of highly separated layers.

The simplest model, the Zero Equation, shows how the air flow gets out of the tube, turns and quickly fits to the down left (as seen in figure 4) corner of the gallery, which will not leave for the complete length of the gallery. The k-epsilon model shows similar behaviour (not considering turbulence effects close to the FRENTE) up to the end of the studied domain (around 25 meters). At that distance the flow turns to the right for 4 meters and then again to the left. The same effect is seen in case of the Spallart-Allmaras model, but this turn is seen closer to the FRENTE, at 16 meters from it.

As we will see later on in the cross sections the Spallart-Allmaras is the best fit, as the experimental data section at 18 meters clearly shows the flow going by the right side of the gallery.

\subsection{Detailed comparison}

\subsubsection{Cross sections}

Data available at $200 \mathrm{~cm}$ from the gallery end will be compared now (see figure 5). All figures, including the experimental data show a highly negative area (blue), in the output of the tube, and a positive area (red) that indicates output flow towards the beginning of the gallery. The intermediate areas (yellows or white in B/W prints) show the area where there is a change in the sense of the flow, the separated flow. 
118 Advances in Fluid Mechanics VI

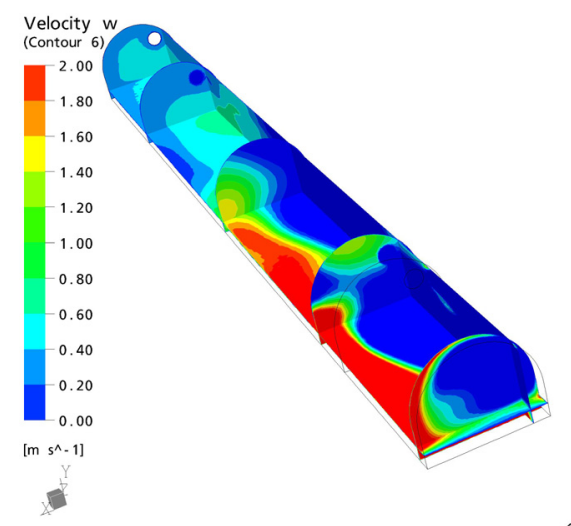

\section{CFE⿱}
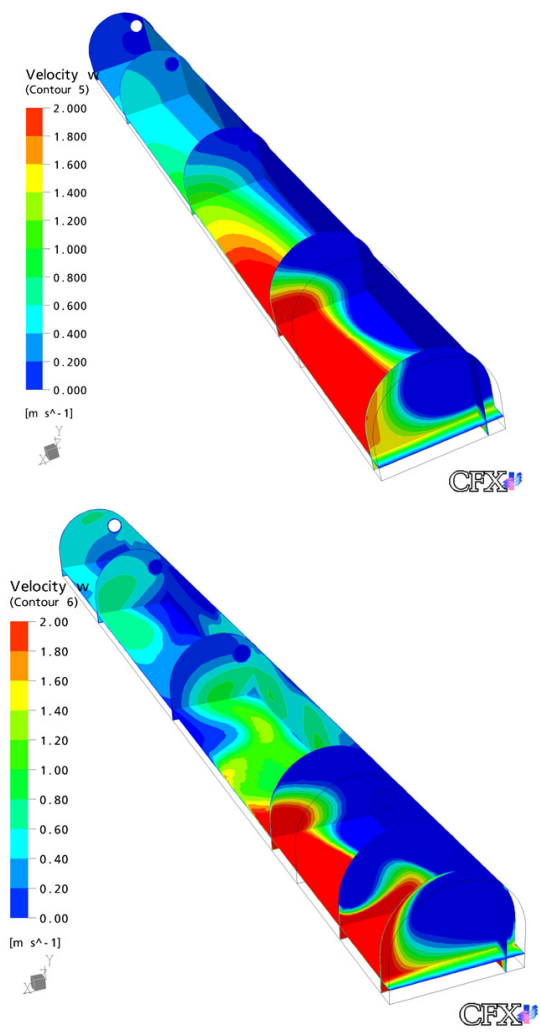

Figure 4: Velocity contours, K-E (up), Zero Equation (middle) and SpalartAllmaras (down). 

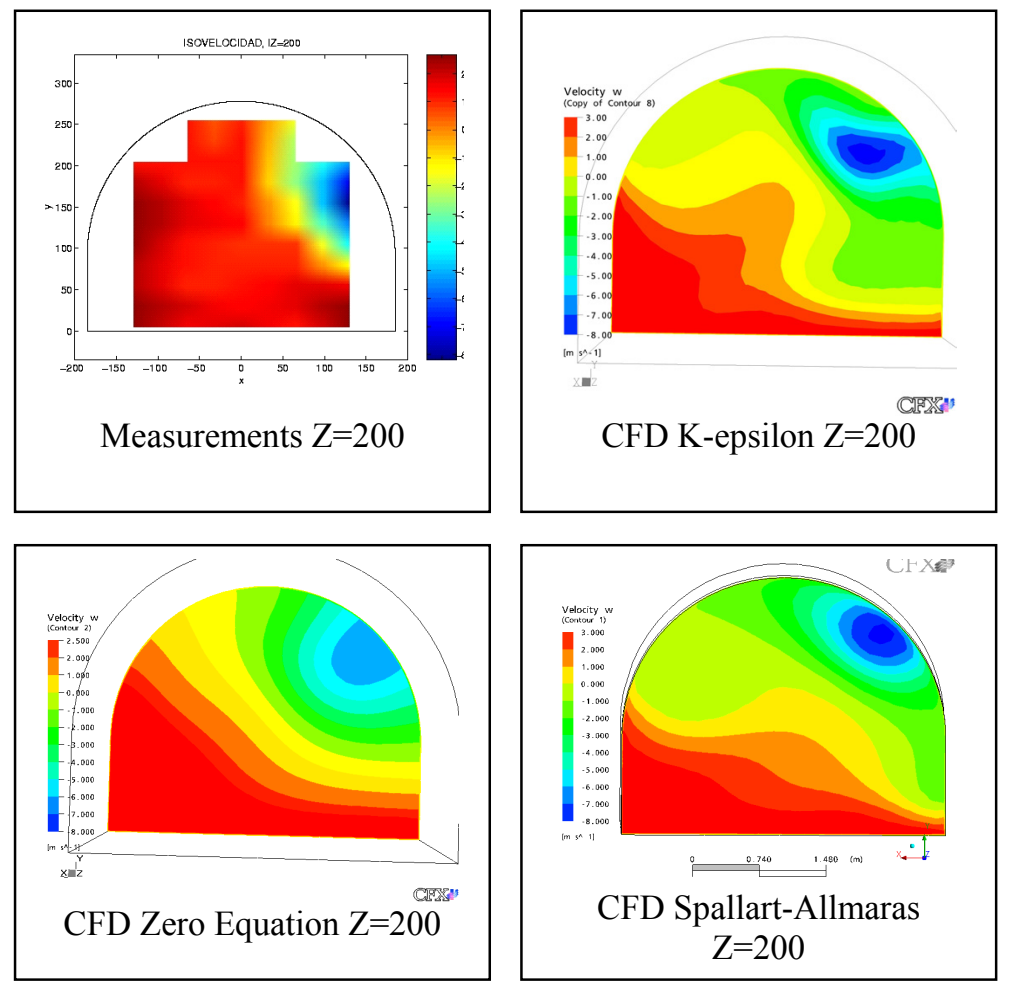

Figure 5: Velocity contours at $Z=200 \mathrm{~cm}$.

In all cases the left down area has positive velocities ranging from 0 to $2 \mathrm{~m} / \mathrm{s}$, occupying a wide area of the bottom. Experimental data shows this area to be very wide, best reflected by the k-epsilon model.

The area immediately in front of the tube output shows a highly negative velocity, again best simulated by the k-epsilon model.

The middle of the gallery shows an experimental velocity between 0 and $1 \mathrm{~m} / \mathrm{s}$. This area is again well predicted by the k-epsilon model.

In any case differences between k-epsilon and Spallart-Allmaras models are not important.

Figure 6 shows equivalent velocity contours in XY planes at 18 meters from gallery end. We can see in the upper left image, the experimental data, that the airflow is now fully developed and velocity values are all below $1 \mathrm{~m} / \mathrm{s}$. The output flow has displaced clearly to the right of the gallery, below the ventilation pipe, and at a medium height. Values below zero, flow towards the gallery end, only appears in the upper part of the gallery, over the ventilation tube.

The only model that predicts this effect is the Spallart-Allmaras, with a $1 \mathrm{~m} / \mathrm{s}$ velocity area in the middle of the gallery, clearly displaced to the right of the image, as in the reality. Both k-epsilon and Zero Equation show the flow in the 
left, stuck to the wall, although k-epsilon shows a more developed flow with almost no areas with velocity below $0,1 \mathrm{~m} / \mathrm{s}$.

This effect can be also clearly seen in figure 4, analysis completed with the fact already commented that the flow turn to the right shown at 18 meters is only seen in case of the k-epsilon model at 25 meters from the gallery end.
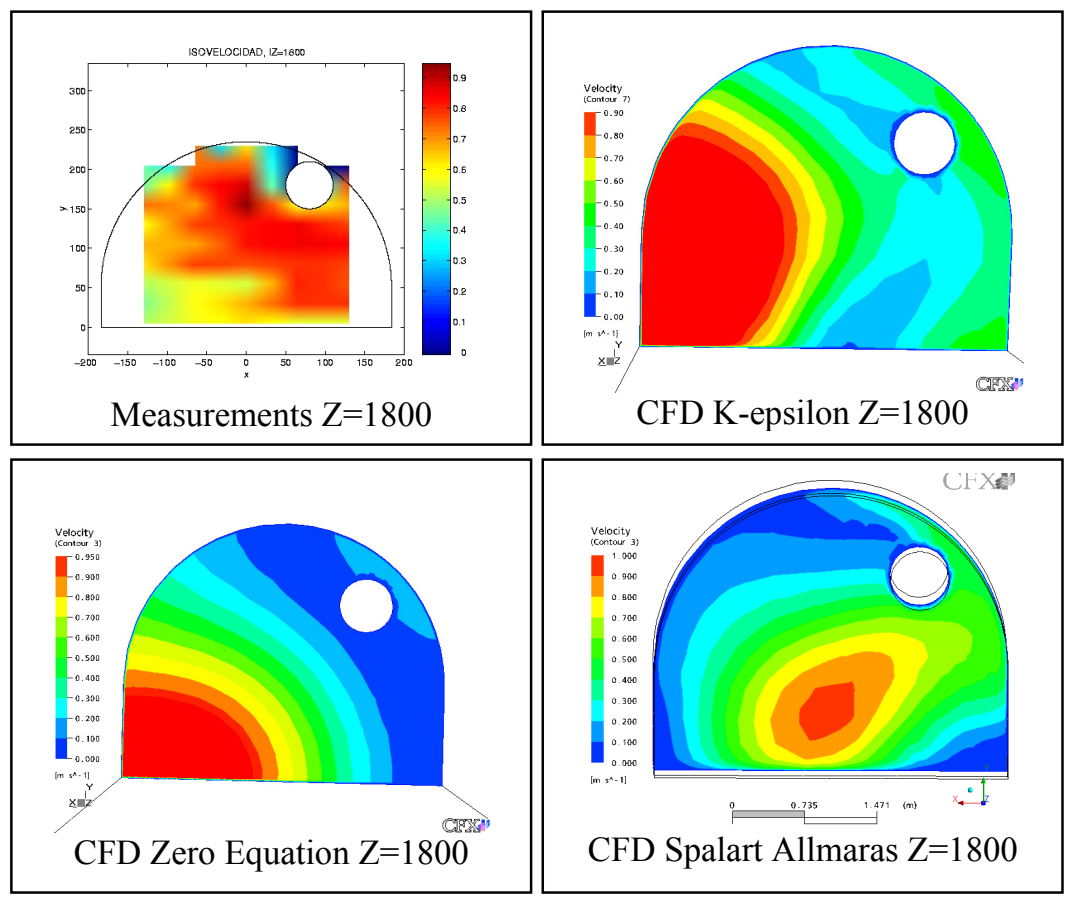

Figure 6: Velocity contours at $Z=1800$.

\section{Conclusions}

Air flow in a death end gallery can be adequately calculated using CFD methods, serving as a base to the injection of particles to simulate dust behaviour in this environment.

The comparison of the results shows good agreement between the experimental data and the simulations done using the Spallart-Allmaras model in the majority of the calculation domain. The area close to the gallery end is best simulated using the k-epsilon model.

The flow makes a characteristic turn around 16 meters from the FRENTE and this turn is simulated in that point by the Spallart-Allmaras model. The K-epsilon model also simulates this turn, but at 25 meters of distance. The simplest model, the Zero Equation, or constant turbulent eddy viscosity model, does not simulate this separation at all, as was expected by the advices referring to its limited use contained in the CFX documentation. 
After the successful velocity field simulation, currently we are developing the study of the evolution of the particulated material inside this airflow, as well as the subsequent comparison with the experimental data obtained by means of optical particle samplers.

We want to acknowledge the help and advices from the Ansys CFX Technical Support Team in the development of these studies.

\section{References}

[1] CFD Help, ANSYS CFX-Solver, Release 10.0: Modelling, pp 98.

[2] K.W. Moloney, I.S. Lowndes and G.K. Hargrave, "Analysis of flow patterns in drivages with auxiliary ventilation", Trans. Instn Min. Metall. 108, pp 17-26 (1999).

[3] A. Wala, J. Jacob, J. Brown and G. Huang, "New approaches to mine-face ventilation", Mining engineering, March 2003, pp 25-30. (2003).

[4] K.W. Moloney and I.S. Lowndes, "Comparison of measured underground air velocities and air flows simulated by computational fluid dynamics", Trans. Instn Min. Metall. 108, pp 105-114 (1999).

[5] J. Toraño, R. Rodriguez, I. Diego and A. Pelegry, "Contamination by particulated material in blasts: analysis, application and adaptation of the existent calculation formulas and software". Environmental Health Risk III, pp. 209-219, (2004).

[6] J. Toraño, R. Rodriguez, J.M. Rivas and A. Pelegry, "Diminishing of the dust quantity during the management of granular material in an underground space", XII International Conference on Modelling Monitoring and management of Air Pollution. (2004).

[7] S.A. Silvester, I.S. Lowndes and S.W. Kingman, "The ventilation of an underground crushing plant", Mining Technology (Trans. Inst. Min. Metall. A), Vol. 113, pp. 201-214 (2004).

[8] López Muñiz, Luis Manuel, PhD. "Explosion retaining through active water barriers", Universidad de Oviedo. 2004. 